\title{
BMJ Open Effectiveness of school-based smoking prevention curricula: systematic review and meta-analysis
}

\author{
Roger E Thomas, ${ }^{1}$ Julie McLellan, ${ }^{2}$ Rafael Perera ${ }^{2}$
}

To cite: Thomas RE,

McLellan J, Perera R.

Effectiveness of school-based smoking prevention curricula: systematic review and metaanalysis. BMJ Open 2015;5: e006976. doi:10.1136/ bmjopen-2014-006976

- Prepublication history and additional material is available. To view please visit the journal (http://dx.doi.org/ 10.1136/bmjopen-2014006976).

Received 22 October 2014 Revised 11 December 2014 Accepted 12 January 2015

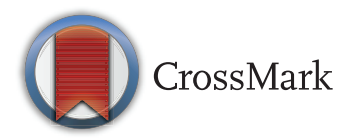

${ }^{1}$ Department of Family Medicine, Faculty of Medicine, University of Calgary, Health Sciences Centre, 3330 Hospital Drive NW, Calgary, Alberta, Canada ${ }^{2}$ Nuffield Department of Primary Care Health Sciences, University of Oxford, Oxford, UK

Correspondence to Professor Roger E Thomas; rthomas@ucalgary.ca

\section{ABSTRACT}

Objective: To assess effectiveness of school-based smoking prevention curricula keeping children neversmokers.

Design: Systematic review, meta-analysis. Data: MEDLINE (1966+), EMBASE (1974+), Cinahl, PsycINFO (1967+), ERIC (1982+), Cochrane CENTRAL, Health Star, Dissertation Abstracts, conference proceedings. Data synthesis: pooled analyses, fixed-effects models, adjusted ORs. Risk of bias assessed with Cochrane Risk of Bias tool.

Setting: 50 randomised controlled trials (RCTs) of school-based smoking curricula.

Participants: Never-smokers age 5-18 ( $n=143495)$; follow-up $\geq 6$ months; all countries; no date/language limitations.

Interventions: Information, social influences, social competence, combined social influences/competence and multimodal curricula.

Outcome measure: Remaining a never-smoker at follow-up.

Results: Pooling all curricula, trials with follow-up $\leq 1$ year showed no statistically significant differences compared with controls (OR 0.91 (0.82 to 1.01)), though trials of combined social competence/social influences curricula had a significant effect on smoking prevention (7 trials, OR $0.59(95 \% \mathrm{Cl} 0.41$ to 0.85$)$ ). Pooling all trials with longest follow-up showed an overall significant effect in favour of the interventions (OR 0.88 (0.82 to 0.95)), as did the social competence (OR 0.65 (0.43 to 0.96)) and combined social competence/social influences curricula (OR 0.60 (0.43 to 0.83$)$ ). No effect for information, social influences or multimodal curricula. Principal findings were not sensitive to inclusion of booster sessions in curricula or to whether they were peer-led or adult-led. Differentiation into tobacco-only or multifocal curricula had a similar effect on the primary findings. Few trials assessed outcomes by gender: there were significant effects for females at both follow-up periods, but not for males.

Conclusions: RCTs of baseline never-smokers at longest follow-up found an overall significant effect with average $12 \%$ reduction in starting smoking compared with controls, but no effect for all trials pooled at $\leq 1$ year. However, combined social competence/social influences curricula showed a significant effect at both follow-up periods.

\section{Strengths and limitations of this study}

- This review and meta-analysis provides evidence from 50 randomised controlled trials with 143495 participants. Comprehensive searches with no limits on data and language mean that it is unlikely trials were missed.

- Using smoking outcomes from cohorts of baseline never-smokers provides the clearest indication of whether smoking prevention curricula are effective.

- Statistical heterogeneity between the trials was low and results were consistent after various sensitivity analyses.

- Not all trials reported outcomes based on cohorts of baseline never-smokers and though authors were contacted it is possible that the data may be incomplete.

- The complexity and reporting of some curricula can make them difficult to classify and therefore the classification of curricula may not be completely accurate.

Systematic review registration: Cochrane Tobacco Review Group CD001293.

\section{INTRODUCTION}

Tobacco use is the main preventable cause of death and disease worldwide, and a global average of $50 \%$ of young males and $10 \%$ of young females start smoking. ${ }^{1}$ It is estimated that smoking will kill about one billion people in the 21st century. ${ }^{1}$ Mortality among smokers is 2-3 times higher than neversmokers and smoking causes a loss of 10 years of life. ${ }^{1}$

In the USA, it has been estimated that of those children who were 17 or younger in 1995 , five million would die prematurely of tobacco-related causes, and that $20 \%$ of deaths could be avoided if smokers had either never started or had quit. ${ }^{2}$ In 2007 , 
in the USA, $20 \%$ of high school students reported smoking in the past 30 days, ${ }^{3}$ and in the UK, the prevalence figures report a smoking rate of $6 \%$ within the 1115 age group. ${ }^{4}$ Starting smoking usually leads to the behaviour lasting decades, with smokers having great difficulty in quitting. Villanti $e t a \check{l}$ identified five types of smoking behaviour as adolescents become young adults: non-smokers, early stable smokers, late starters, quitters and 'light or intermittent smokers'.

Over the past three decades, the school environment has been a particular focus of efforts to influence youth smoking behaviour. The main perceived advantages are that almost all children can be reached through schools and a focus on tobacco education fits naturally within their daily activities. Researchers have used five types of curriculum in schools, each based on a different theoretical orientation: information-only curricula, social competence curricula, social influence curricula, combined social competence/social influences curricula and multimodal curricula ${ }^{6}$ (box 1).

Social competence interventions help adolescents refuse offers to smoke by improving their general social competence and personal and social skills. Adolescents are taught a combination of skills to improve problem solving, decision-making, self-control, self-esteem, assertiveness and strategies to cope with stress, and to resist general personal or media influences.

Social influence interventions focus specifically on teaching adolescents skills for awareness of social influences that encourage substance use, and to resist

Box 1 Types of curricula in schools to prevent smoking

\section{Information only curricula}

Interventions that provide information to correct inaccurate perceptions regarding the prevalence of tobacco use and oppose inaccurate beliefs that smoking is social acceptable.

Social competence curricula

Interventions that help adolescents refuse offers to smoke by improving their general social competence and personal and social skills. Interventions teach problem solving, decisionmaking, cognitive skills to resist personal or media influences, increase self-control and self-esteem, coping strategies for stress and assertiveness skills.

Social influence curricula

Interventions that endeavour to overcome social influences to use tobacco by teaching adolescents to be aware of social influences that encourage substance use, teach skills to resist offers of tobacco, and deal with peer pressure and high-risk situations that might persuade an adolescent directly or indirectly to smoke. Combined social competence and social influences curricula Multimodal curricula

Programmes in schools and the community, involving parents and community members, initiatives to change school or state policies about tobacco sales and taxes, and to prevent sales to minors.

Other

School antismoking policies, motivations to smoke, classroom good behaviour. tobacco offers, peer pressure and high risk situations that might persuade an adolescent directly or indirectly to smoke. Some studies have tested teaching skills to resist multiple problem behaviours such as drinking and drug use as well as tobacco use. Multimodal interventions can be broad ranging, including tobacco prevention interventions in schools, the community, and with parents and community members, and school or state policies to change tobacco sales, increase taxes and prevent sales to minors.

The first edition of this Cochrane review was published in 2002, included 96 studies and was narrative without any meta-analyses. The second edition incorporated meta-analyses for randomised controlled trials (RCTs) with relevant information for smoking prevention, but the largest comparison only contained 13 studies. Authors often include data for never-smokers, triers, quitters, occasional, regular and heavy smokers in their baseline and follow-up data. Some use the term current 'non-smokers' and include never-smokers, triers, experimenters and quitters. It is thus not possible to determine the effect of smoking prevention curricula interventions on each of these groups, and if some groups increased and others decreased their smoking, the effect of the curricula could be completely obscured. We were thus able to prespecify that the ideal outcome to give the best estimate of the prevention effect would be baseline neversmoking cohorts, and were then able to extract more evidence from existing and new studies without changing the curricula classification in the review protocol.

Hence, in 2013, the second edition was updated and radically refined: we checked the theoretical orientation of each trial and all included trials were recategorised, and data completely re-extracted and reanalysed based on baseline never-smoking cohorts. The primary objective of this review is to assess the effectiveness of school-based curricula versus no curricula in preventing never-smoking children and adolescents from starting smoking. Effectiveness is the appropriate term as researchers tested interventions in real schools, but did not always control for adherence or attendance. A second objective is to assess which curricula types are the most effective.

\section{METHODS}

\section{Search strategy and trial selection}

We searched the Cochrane Central Register of Controlled Trials (CENTRAL), the Cochrane Tobacco Addiction Group's Specialized Register, MEDLINE, EMBASE, PsyclNFO, ERIC, CINAHL, Health Star and Dissertation Abstracts for terms relating to school-based smoking cessation programmes from inception to January 2014, with no date or language restrictions (see online supplementary material A). We checked article bibliographies and ran individual MEDLINE searches for 133 authors who had undertaken research in this area. We searched for all trials evaluating school-based 
curricula to prevent smoking. There was no restriction on the theoretical orientation of the curricula providing they aimed to prevent tobacco use. Students aged 5-18 years during the intervention phase of the trial were included as individuals in RCTs and also as classes, schools or school districts in cluster RCTs (C-RCTs). Trials were excluded if there was no control group. Control groups included no curricula, usual practice or an active non-relevant control, for example, homework study group. We required a minimum follow-up of 6 months after completion of the curricula. We did not require biochemical validation of self-reported tobacco use, but recorded its use. We excluded trials that did not assess baseline smoking status or reported only smoking attitudes and knowledge.

Two reviewers (RET and JM) independently assessed all titles, abstracts and full text articles for trials that met the inclusion criteria. Any disagreements were resolved by consensus or referral to a third person (RP).

\section{Data extraction and study classification}

Data were independently extracted into RevMan ${ }^{7}$ by two reviewers (RET and JM) for each included study using a form piloted first in a small subset of trials. Any disagreements were resolved by consensus or referral to the third author (RP). We extracted data for all included trials on design and focus, country and site of school(s), participants (age, gender and ethnicity), curriculum duration and follow-up, curriculum deliverer, a brief overview of the curriculum, and details of the control group. Two authors (RET and JM) classified curricula according to their dominant theoretical orientation: information only, social competence, social skills, combined social competence/social skills or multimodal. An independent reviewer commented on this classification and as a result a small number of trials using strategies that did not fit into these broad types were grouped separately (box 1). Accuracy of category classification between the authors and the independent reviewer was tested using a $\mathrm{\kappa}$ statistic.

We extracted data for never-smokers at baseline and follow-up for curricula and control groups. If authors included in the category of 'non-smoker' both neversmokers and those not currently smoking, we classified non-smokers with previous smoking experience as smokers for this review.

We used the Cochrane risk of bias $\operatorname{tool}^{8}$ to assess whether trials were at low, high or unclear risk of selection bias (random sequence generation and allocation concealment), detection bias, attrition bias and reporting bias.

If data were missing, or in a format not analysable, we contacted the authors to request the data, new data runs or clarification. We did not impute missing data.

\section{Data analysis}

We extracted data as absolute numbers or ORs, where possible, based on loss of never-smokers from baseline to follow-up, that is, those children who started smoking. In some instances, if data were available, but only the total number of schools or classes was known and not the numbers allocated to each arm, then the number of schools or classes was estimated based on the proportion of individuals within the group. Where the authors used a denominator that did not include all the participants originally randomised (eg, a sample that the author described as the 'analysis sample,' which excluded dropouts and thus had smaller numbers at follow-up) we recomputed the data based on the same percentage loss to never-smokers using the numbers originally randomised. We calculated adjusted ORs based on the number of never-smokers at specific time points. Adjustment was made for clustering by school/group based on estimated intraclass correlation coefficients (0.097) and cluster sizes to determine design effects for each of the curricula groups. We then used this design effect to determine the effective sample size for each curricula group.

Our analysis used a fixed effects meta-analysis using the generalised inverse variance method. Only trials for which never-smoking outcome data could be extracted were included in the analysis. Trial data were excluded if the publication or author could not provide data or the data were incomplete for either the curricula or control groups for baseline or follow-up, where the number of cluster sizes could not be extracted or estimated, where the data were in an unusable format or where the data were judged to be unreliable or contradictory. The included data were pooled to obtain estimates for an overall effect, with subgroups based on curriculum used. Trials in the 'other curricula' group were sufficiently different from each other so that, although they were presented within the meta-analysis for the entire group, it would be inappropriate to combine them as a distinct group by curriculum within the Results and Discussion sections. If a trial compared more than one curriculum arm then the control group was split equally between the arms for outcome events as well as sample size. We used the $\mathrm{I}^{2}$ statistic to assess inconsistency across trials and provide a measure of heterogeneity. ${ }^{9}$

Our analysis examined the curricula versus the control groups at two defined times of follow-up: 1 year or less and longest follow-up. In the latter, we used one set of data at the longest follow-up point for each study, meaning that some data sets appeared in both analyses. In order to determine the impact that trials only reporting short-term follow-up (1 year or less) had on our long-term effect estimates, we carried out a sensitivity analysis excluding these studies from this estimate.

A priori we identified attrition and selection as the two most relevant sources of bias. We conducted sensitivity analyses to compare the overall result of trials with low risk of attrition and selection bias to all trials to see whether the quality of the trials had any impact on the overall results. Risk of publication bias was assessed by a visual inspection of a funnel plot. 
We further conducted subanalyses based on gender, peer-led (or substantially peer-led) versus adult-led trials, trials with a tobacco-only focus (tobacco-only) versus multifocal curricula (curricula that focused on tobacco together with other substances such as alcohol and drugs), and curricula that had subsequent booster sessions versus those with none. Booster sessions were additional 'refresher' sessions separate from the initial curricula. Though not prespecified, we subsequently explored whether it was relevant to complete a subanalysis by age (age 11 and under vs over 11).

\section{RESULTS}

We identified 256 potential RCTs or C-RCTs. Of these, 135 C-RCTs and 1 RCT provided a total of 202 different curricula arms with 431315 participants providing data (figure 1). Trials were categorised by curricula type; the robustness of this classification was confirmed as very good when the agreement between authors and an independent reviewer was tested $(\kappa 0.98)$.

Fifty-seven of the 136 trials followed never-smoking cohorts and of these 50 C-RCTs (74 different intervention arms, $\mathrm{n}=143495)$ provided analysable data for this review (figure 1 ).

\section{Characteristics of included studies}

Table 1 provides a summary of the baseline characteristics of included studies. The control groups in the 50 trials were varied. In $22(44 \%)$, the group receiving the curriculum was compared head-to-head with a control group that received 'usual practice,' in 12 trials the control group received no alternative curricula, nine did not state whether the control group received an alternative curricula, 1 provided no alternative curriculum in the control group in six schools and 'usual practice' in the control group in four schools, 2 provided only information, 1 provided a curriculum to help students complete schoolwork, 1 offered a talk by a physician on either tobacco or alcohol, 1 posted four booklets to the control group, 1 asked students to produce a newspaper and 1 helped students with reading skills. Of the 50 trials, 47 were in individual countries and 3 in multiple countries (total 60 country arms): 26 trials were from the USA, 4 each from the UK, Netherlands and Germany, 3 from each from Spain and Italy, 2 each from Australia, Canada and China and the remainder 1 each from South Africa, Thailand and across Europe (Denmark, Finland, Portugal, Austria, Belgium, Greece, Sweden and the Czech Republic).

\section{Principal findings}

(See online supplementary material B for raw data).

All curricula types versus control, with follow-up 1 year or less (26 trials, 41 curriculum arms, figure 2 and table 2 )

There was no overall effect for all curricula with follow-up of 1 year or less (OR $0.91,95 \%$ CI 0.82 to 1.01 ; $\left.I^{2}=19 \%\right)$. The $I^{2}$ statistic for subgroup differences across all curricula was $45.9 \%$, but within each curriculum type heterogeneity was minimal, except for multimodal $\left(\mathrm{I}^{2}=51 \%\right)$. The combined social competence/social influences curricula (seven C-RCTs/eight arms) showed a statistically significant effect in preventing the onset of smoking at 1 year or less (OR 0.59 , CI 0.41 to $\left.0.85 ; \mathrm{I}^{2}=0 \%\right)$. However, for the social influences curricula (16 RCTs/25 arms), the multimodal curricula (3 RCTs/5 arms) and 1 small trial, ${ }^{36}$ which tested an information-only curriculum, the results were non-significant. There was no RCT testing a social competence curriculum versus control with a follow-up duration of 1 year or less.

All curricula types versus control had the longest follow-up (50 trials, 74 curriculum arms, figure 3 and table 2).

Fifteen trials (25 arms) provided data for analysis at follow-up of 1 year or less and for longest follow-up (34\% of trials). Of the remaining trials, $86 \%$ had follow-up of between 1 and 5 years, $10 \%$ of between 5 and 10 years and $4 \%$ of over 10 years.

There was a significant effect favouring all curricula compared with control for the longest follow-up periods (OR $0.88,95 \%$ CI 0.82 to $0.95 ; \mathrm{I}^{2}=12 \%$ ), with a mean risk reduction of $12 \%$. Heterogeneity was low $(0-12 \%)$, except for the multimodal curricula trials $\left(I^{2}=64 \%\right)$.

Our estimate of long-term effect was robust to the exclusion of trials that reported only short-term (1 year or less) follow-up (see online supplementary material C). There were 10 trials ( 15 arms) that provided separate data both for analysis at 1 year or less and for the analysis at longest follow-up. Restricting the analysis to these trials alone showed the same overall effects as the primary findings, no overall effect at 1 year or less follow-up and a statistically significant effect at longest follow-up.

By individual curricula, social competence curricula (5 C-RCTs/7 arms) compared with control showed a statistically significant result in favour of the curricula (OR 0.65 , CI 0.43 to $0.96 ; \mathrm{I}^{2}=0 \%$ ) and also the combined social competence/social influences (9 C-RCTs/ $11 \mathrm{arms}$ ) compared with control (OR 0.60, CI 0.43 to $\left.0.83 ; \mathrm{I}^{2}=0 \%\right)$. There were no statistically significant differences for the one information-only curriculum, or the social influences or multimodal curricula. Four trials (six arms) were classified as 'other curricula' and contributed to the overall results, but not to the individual curricula types. ${ }^{1732} 3839$

\section{Sensitivity analysis}

(See online supplementary material D for sensitivity analyses).

Sensitivity analyses restricted to trials at low risk of attrition bias with follow-up of 1 year or less $(n=9)$ found no differences compared with all trials in terms of point estimates, though trials testing combined social competence/ social influences curricula no longer demonstrated a significant effect when studies at unclear or high risk of bias were removed (OR 0.55 , CI 0.28 to 1.09 ). At longest 
Search $2006-2014$ updates

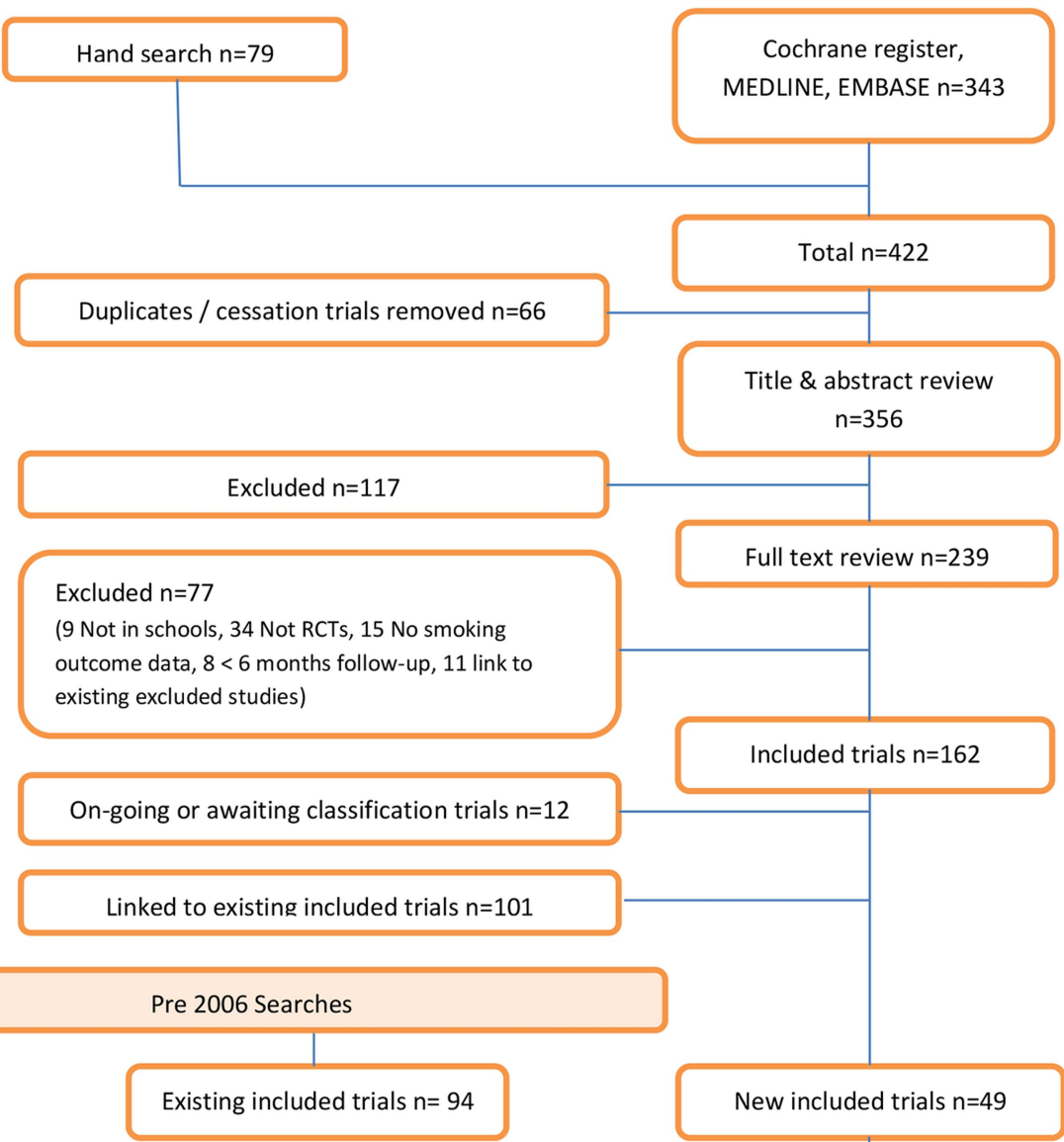

Re-classification of trials for 2014 new analysis

Excluded on re-examination $\mathrm{n}=7$ (3 Not RCTS, 1 Not in schools, 2 No smoking outcome data, 1 linked to existing trial)

Data only available as changes in smoking behaviour over time $n=6$

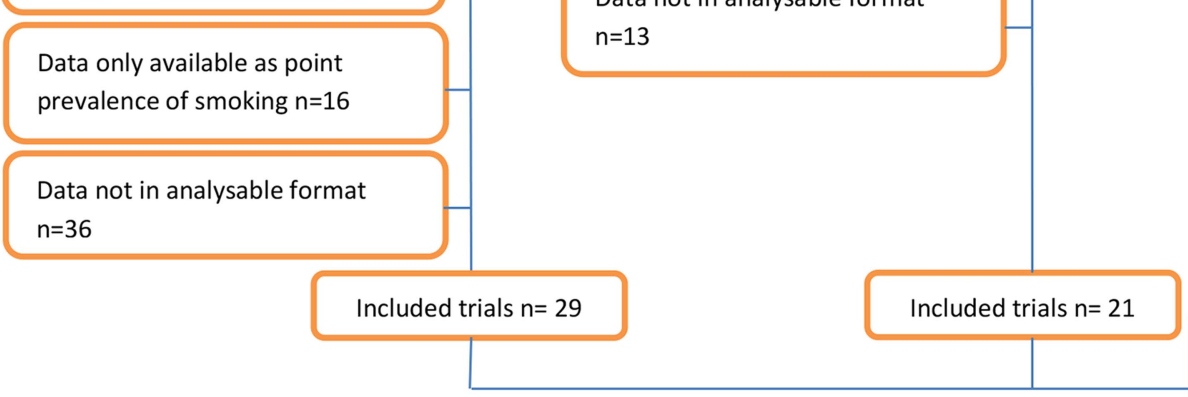

Included trials (baseline never smoking cohorts) $n=50$

Figure 1 Flow diagram to show selection process (RCTs, randomised controlled trials).

follow-up, analyses restricted to low risk of attrition bias $(n=20)$ were similar to pooled results from all trials, except the CI was wider and hence included the line of no effect for trials at low risk of bias (OR 0.90, CI 0.80 to 1.03) compared with all trials (OR 0.88, CI 0.82 to 0.95$)$. 


\begin{tabular}{|c|c|c|c|c|c|c|c|c|c|c|}
\hline Study name & $\begin{array}{l}\text { Alternative name } \\
\text { (if applicable) }\end{array}$ & $\begin{array}{l}\text { Study } \\
\text { design }\end{array}$ & $\begin{array}{l}\text { Average } \\
\text { age } \\
\text { (years) }\end{array}$ & $\begin{array}{l}\text { Gender } \\
\% \text { female }\end{array}$ & $\begin{array}{l}\text { Curriculum } \\
\text { intensity } \\
\text { (sessions) }\end{array}$ & $\begin{array}{l}\text { Curriculum } \\
\text { duration (months, } \\
\text { unless otherwise } \\
\text { stated) }\end{array}$ & $\begin{array}{l}\text { Curriculum } \\
\text { deliverer }\end{array}$ & $\begin{array}{l}\text { Control group } \\
\text { type }\end{array}$ & $\begin{array}{l}\text { Ethnicity } \\
\text { (dominant) }\end{array}$ & Country \\
\hline $\begin{array}{l}\text { Armstrong et al } \\
\text { (peer) }^{10}\end{array}$ & & C-RCT & 12 & 49 & 5 & 6 & Peers & No curriculum & NS & Australia \\
\hline $\begin{array}{l}\text { Armstrong et al } \\
\text { (teacher) }\end{array}$ & & C-RCT & 12 & 49 & 5 & 6 & Teachers & No curriculum & NS & Australia \\
\hline $\begin{array}{l}\text { Ausems et al } \\
\text { (in school) }^{11}\end{array}$ & & C-RCT & 13 & 52 & $3 \times 50 \min$ & NS & Teachers & NS & NS & The Netherlands \\
\hline $\begin{array}{l}\text { Ausems et al } \\
\text { (out school) }^{11}\end{array}$ & & C-RCT & 13 & 52 & NS & NS & Teachers & NS & NS & The Netherlands \\
\hline $\begin{array}{l}\text { Aveyard } \\
\text { et al1999 }\end{array}$ & & C-RCT & 13.5 & 50 & $6 \times 1 \mathrm{~h}$ & 12 & Teachers & Usual practice & $86 \%$ White & UK \\
\hline $\begin{array}{l}\text { Botvin and Eng } \\
1980^{13}\end{array}$ & & C-RCT & 13.5 & NS & 10 & 3 & $\begin{array}{l}\text { Outside } \\
\text { specialists }\end{array}$ & No curriculum & White & USA \\
\hline $\begin{array}{l}\text { Botvin and Eng } \\
1982^{14}\end{array}$ & & C-RCT & 12.5 & NS & $12 \times 1 \mathrm{~h}$ & 3 & Peers & No curriculum & $90 \%+$ White & USA \\
\hline $\begin{array}{l}\text { Botvin et al } \\
\text { (LST intensive) }^{15}\end{array}$ & & C-RCT & 12.5 & NS & 15 & 1 & Teachers & Usual practice & $91 \%$ White & USA \\
\hline $\begin{array}{l}\text { Botvin et al } \\
\text { (LST) }^{15}\end{array}$ & & C-RCT & 12.5 & NS & 15 & 3.5 & Teachers & Usual practice & $91 \%$ White & USA \\
\hline Botvin et al ${ }^{16}$ & & C-RCT & 11.5 & 100 & $15+10$ boosters & NS & Teachers & $\begin{array}{l}10 \text { sessions of } \\
\text { information only, } \\
\text { plus } 3 \text { boosters }\end{array}$ & $\begin{array}{l}60 \% \\
\text { African-American }\end{array}$ & USA \\
\hline Brown et al ${ }^{17}$ & & C-RCT & 13.5 & 50 & NS & NS & $\begin{array}{l}\text { Students and } \\
\text { teachers }\end{array}$ & Usual practice & NS & Canada \\
\hline $\begin{array}{l}\text { Buller et al } \\
\text { (Australia) }^{18}\end{array}$ & Consider This & C-RCT & 11 to 14 & 52 & $6 \times 1 \mathrm{~h}$ & 6 & Web-based & Usual practice & $\begin{array}{l}73 \% \text { Australian/ } \\
\text { European }\end{array}$ & Australia \\
\hline $\begin{array}{l}\text { Buller et al } \\
(\text { USA })^{18}\end{array}$ & Consider This & C-RCT & 11 to 13 & 52 & $6 \times 1 \mathrm{~h}$ & 6 & Web-based & Usual practice & $56 \%$ White & USA \\
\hline Chou et a $1^{19}$ & & C-RCT & 12.5 & 48 & $13 \times 45 \mathrm{~min}$ & 3 & $\begin{array}{l}\text { Health } \\
\text { educators } \\
\text { (USA) }\end{array}$ & Usual practice & NS & China \\
\hline Coe et $a P^{\circ}$ & & C-RCT & 12.5 & NS & 8 & NS & $\begin{array}{l}\text { Medical } \\
\text { students }\end{array}$ & No curriculum & $88 \%+$ White & USA \\
\hline Connell et $a P^{11}$ & $\begin{array}{l}\text { Adolescent } \\
\text { Transitions } \\
\text { Programme }\end{array}$ & C-RCT & 11 & 47 & 6 & 2 & $\begin{array}{l}\text { Parent } \\
\text { consultants }\end{array}$ & NS & $42 \%$ White & USA \\
\hline $\begin{array}{l}\text { Conner and } \\
\text { Higgins }(I)^{22}\end{array}$ & & C-RCT & 11.5 & 50 & NS & 24 & NS & $\begin{array}{l}\text { Information and } \\
\text { homework } \\
\text { intentions }\end{array}$ & NS & UK \\
\hline Crone et $a f^{33}$ & & C-RCT & 10 to 12 & 53 & $6 \times 1 \mathrm{~h}$ & 24 & Teachers & Usual practice & NS & The Netherlands \\
\hline $\begin{array}{l}\text { De Vries et al } \\
(\text { High })^{24}\end{array}$ & & C-RCT & 12.5 & NS & $5 \times 45 \min$ & NS & $\begin{array}{l}\text { Peers and } \\
\text { teachers }\end{array}$ & NS & NS & The Netherlands \\
\hline $\begin{array}{l}\text { De Vries et al } \\
\text { (Denmark) })^{25}\end{array}$ & $\begin{array}{l}\text { European Smoking } \\
\text { Prevention } \\
\text { Framework } \\
\text { Approach }\end{array}$ & C-RCT & 13 & 50 & $6 \times 1 \mathrm{~h}$ & NS & Teachers & Usual practice & European & Denmark \\
\hline
\end{tabular}




\begin{tabular}{|c|c|c|c|c|c|c|c|c|c|c|}
\hline Study name & $\begin{array}{l}\text { Alternative name } \\
\text { (if applicable) }\end{array}$ & $\begin{array}{l}\text { Study } \\
\text { design }\end{array}$ & $\begin{array}{l}\text { Average } \\
\text { age } \\
\text { (years) }\end{array}$ & $\begin{array}{l}\text { Gender } \\
\% \text { female }\end{array}$ & $\begin{array}{l}\text { Curriculum } \\
\text { intensity } \\
\text { (sessions) }\end{array}$ & $\begin{array}{l}\text { Curriculum } \\
\text { duration (months, } \\
\text { unless otherwise } \\
\text { stated) }\end{array}$ & $\begin{array}{l}\text { Curriculum } \\
\text { deliverer }\end{array}$ & $\begin{array}{l}\text { Control group } \\
\text { type }\end{array}$ & $\begin{array}{l}\text { Ethnicity } \\
\text { (dominant) }\end{array}$ & Country \\
\hline $\begin{array}{l}\text { De Vries et al } \\
\text { (Finland) }^{25}\end{array}$ & $\begin{array}{l}\text { European Smoking } \\
\text { Prevention } \\
\text { Framework } \\
\text { Approach }\end{array}$ & $\mathrm{C}-\mathrm{RCT}$ & 13 & 50 & $5 \times 45 \min$ & NS & Teachers & Usual practice & European & Finland \\
\hline $\begin{array}{l}\text { De Vries et al } \\
(\text { Portugal) })^{25}\end{array}$ & $\begin{array}{l}\text { European Smoking } \\
\text { Prevention } \\
\text { Framework } \\
\text { Approach }\end{array}$ & $\mathrm{C}-\mathrm{RCT}$ & 13 & 50 & 6 & NS & Teachers & Usual practice & European & Portugal \\
\hline $\begin{array}{l}\text { De Vries et al } \\
(\mathrm{UK})^{25}\end{array}$ & $\begin{array}{l}\text { European Smoking } \\
\text { Prevention } \\
\text { Framework } \\
\text { Approach }\end{array}$ & $\mathrm{C}-\mathrm{RCT}$ & 13 & 50 & $50 \times 30 \mathrm{~min}$ & NS & Teachers & Usual practice & European & UK \\
\hline $\begin{array}{l}\text { Denson and } \\
\text { Stretch }\end{array}$ & & $\mathrm{C}-\mathrm{RCT}$ & 12 to 14 & NS & 3 & 24 & Researcher & No curriculum & NS & Canada \\
\hline Elder et al $1996^{27}$ & $\mathrm{CATCH}$ & $\mathrm{C}-\mathrm{RCT}$ & 10.5 & 51 & $4 \times 50 \mathrm{~min}$ & NS & Teachers & No curriculum & $71 \%$ White & USA \\
\hline $\begin{array}{l}\text { Ellickson and Bell } \\
(\text { HealthEd })^{28}\end{array}$ & ALERT & $\mathrm{C}-\mathrm{RCT}$ & 13.5 & 48 & $8+3$ booster & 2 & $\begin{array}{l}\text { Community } \\
\text { adults }\end{array}$ & $\begin{array}{l}\text { No curriculum or } \\
\text { usual practice }\end{array}$ & $67 \%$ White & USA \\
\hline $\begin{array}{l}\text { Ellickson and Bell } \\
1990 \text { (Teen) })^{28}\end{array}$ & ALERT & $\mathrm{C}-\mathrm{RCT}$ & 13.5 & 48 & $8+3$ booster & 2 & Students & $\begin{array}{l}\text { No curriculum or } \\
\text { usual practice }\end{array}$ & $67 \%$ White & USA \\
\hline Ellickson et $a^{29}$ & ALERT & C-RCT & 12.5 & 50 & $7+3$ & NS & Teachers & Usual practice & NS & USA \\
\hline Ennett et a ${ }^{\beta 0}$ & DARE & $\mathrm{C}-\mathrm{RCT}$ & 10.5 & 49 & $17 \times 1 \mathrm{~h}$ & 4 & $\begin{array}{l}\text { Uniformed } \\
\text { police officer }\end{array}$ & NS & $54 \%$ White & USA \\
\hline Faggiano et $a{ }^{\beta 1}$ & Unplugged & C-RCT & 12 to 14 & 48 & $12 \times 1 \mathrm{~h}$ & 3 & Teachers & Usual practice & NS & $\begin{array}{l}\text { Austria, Belgium, } \\
\text { Germany, Greece, } \\
\text { Italy, Spain, } \\
\text { Sweden }\end{array}$ \\
\hline $\begin{array}{l}\text { Figa-Talamanca } \\
\text { and Modolo }{ }^{32}\end{array}$ & & $\mathrm{C}-\mathrm{RCT}$ & 15 to 17 & 47 & 3 & 3 (days) & $\begin{array}{l}\text { Health } \\
\text { educators }\end{array}$ & No curriculum & NS & Italy \\
\hline Gabrhelik et $a{ }^{\beta 3}$ & Unplugged & C-RCT & 11 & 50 & $12 \times 45 \min$ & 12 & Teachers & Usual practice & Czech & Czech Republic \\
\hline Garcia et al $2005^{34}$ & ALERT & C-RCT & 13 & 47 & $8 \times 1 h$ & NS & Teachers & Usual practice & NS & Spain \\
\hline Hort et $a P^{35}$ & & $\mathrm{C}-\mathrm{RCT}$ & 13 & 38 & $\begin{array}{l}4 \times 1-2 h+ \\
15 \times 1 h\end{array}$ & 24 & $\begin{array}{l}\text { Physicians and } \\
\text { teachers }\end{array}$ & $\begin{array}{l}\text { Physician talk on } \\
\text { smoking if } \\
\text { requested }\end{array}$ & NS & Germany \\
\hline Howard et $a \beta^{\beta 6}$ & & C-RCT & 10 & 46 & $5 \times 40 \min$ & NS & Teachers & NS & NS & USA \\
\hline Johnson et $a l^{37}$ & $\begin{array}{l}\text { Acadiana Coalition } \\
\text { of Teens against } \\
\text { Tobacco }\end{array}$ & C-RCT & 15 & 51 & NS & 30 & Teachers & NS & $61 \%$ White & USA \\
\hline $\begin{array}{l}\text { Kellam and } \\
\text { Anthony }(\mathrm{GBG})^{38}\end{array}$ & $\begin{array}{l}\text { Good Behaviour } \\
\text { Game }\end{array}$ & C-RCT & 5.5 & 50 & $\begin{array}{l}3 \times \text { per } \\
\text { week } \times 10 \mathrm{~min}\end{array}$ & 24 & Teachers & Usual practice & $\begin{array}{l}70 \% \\
\text { African-American }\end{array}$ & USA \\
\hline $\begin{array}{l}\text { La Torre et al } \\
\text { (adolescents) }^{39}\end{array}$ & & $\mathrm{C}-\mathrm{RCT}$ & 14 & 52 & NS & NS & Teachers & NS & NS & Italy \\
\hline $\begin{array}{l}\text { Luna-Adame } \\
\text { et } a f^{40}\end{array}$ & & C-RCT & 11 & 51 & $\begin{array}{l}21 \times 1 \mathrm{~h} \text { in year } 1 \text {, } \\
12 \times 1 \mathrm{~h} \text { in } \\
\text { second year }\end{array}$ & 24 & $\begin{array}{l}\text { Psychology } \\
\text { students }\end{array}$ & Usual practice & NS & Spain \\
\hline
\end{tabular}




\begin{tabular}{|c|c|c|c|c|c|c|c|c|c|c|}
\hline Study name & $\begin{array}{l}\text { Alternative name } \\
\text { (if applicable) }\end{array}$ & $\begin{array}{l}\text { Study } \\
\text { design }\end{array}$ & $\begin{array}{l}\text { Average } \\
\text { age } \\
\text { (years) }\end{array}$ & $\begin{array}{l}\text { Gender } \\
\% \text { female }\end{array}$ & $\begin{array}{l}\text { Curriculum } \\
\text { intensity } \\
\text { (sessions) }\end{array}$ & $\begin{array}{l}\text { Curriculum } \\
\text { duration (months, } \\
\text { unless otherwise } \\
\text { stated) }\end{array}$ & $\begin{array}{l}\text { Curriculum } \\
\text { deliverer }\end{array}$ & $\begin{array}{l}\text { Control group } \\
\text { type }\end{array}$ & $\begin{array}{l}\text { Ethnicity } \\
\text { (dominant) }\end{array}$ & Country \\
\hline $\begin{array}{l}\text { Nutbeam et al } \\
(\mathrm{FSE})^{41}\end{array}$ & & C-RCT & 11.5 & 43 & 3 & NS & Teachers & No curriculum & NS & UK \\
\hline $\begin{array}{l}\text { Peterson et al } \\
2000^{42}\end{array}$ & $\begin{array}{l}\text { Hutchinson } \\
\text { Smoking Prevention } \\
\text { Project }\end{array}$ & C-RCT & 7 to 9 & 49 & 65 & NS & Teachers & Usual practice & $90 \%$ Caucasian & USA \\
\hline $\begin{array}{l}\text { Piper et al (HFL } \\
\text { Age) })^{43}\end{array}$ & $\begin{array}{l}\text { Healthy for Life } \\
\text { Project }\end{array}$ & C-RCT & 14.5 & 52 & $\begin{array}{l}58 \text { (in } 3 \times 4 \text {-week } \\
\text { periods) }\end{array}$ & 36 & $\begin{array}{l}\text { Community } \\
\text { adults }\end{array}$ & Usual practice & $92 \%+$ White & USA \\
\hline Piper et al (HFL) ${ }^{43}$ & $\begin{array}{l}\text { Healthy for Life } \\
\text { Project }\end{array}$ & C-RCT & 14.5 & 52 & 54 & 12 & $\begin{array}{l}\text { Community } \\
\text { adults }\end{array}$ & Usual practice & $92 \%+$ White & USA \\
\hline Prokhorov et $a f^{4}$ & $\begin{array}{l}\text { A Smoking } \\
\text { Prevention } \\
\text { Interactive } \\
\text { Experience }\end{array}$ & C-RCT & 16 & 59 & $\begin{array}{l}5 \times 30 \mathrm{~min}+2 \\
\text { boosters }\end{array}$ & NS & Computer & Usual practice & $51 \%$ Hispanic & USA \\
\hline $\begin{array}{l}\text { Resnicow et al } \\
(\text { Harm Min })^{45}\end{array}$ & Keep Left & C-RCT & 14 & 50 & 8 & 24 & Teachers & Usual practice & $60 \%$ Black & South Africa \\
\hline $\begin{array}{l}\text { Resnicow et al } \\
(\text { LST })^{45}\end{array}$ & Life Skills Training & C-RCT & 14 & 50 & 8 & 24 & Teachers & Usual practice & $60 \%$ Black & South Africa \\
\hline Ringwalt et $a l^{46}$ & ALERT & C-RCT & 11 & 52 & $\begin{array}{l}11 \times 45 \mathrm{~min}+3 \\
\text { boosters }\end{array}$ & 24 & Teachers & No curriculum & $53 \%$ White & USA \\
\hline Schulze et $a{ }^{47}$ & $\begin{array}{l}\text { Be smart-don't } \\
\text { start }\end{array}$ & C-RCT & 12 & 50 & NS & NS & Teachers & No curriculum & NS & Germany \\
\hline Seal $^{48}$ & & C-RCT & 15.5 & 11 & $10 \times 1 \mathrm{~h}$ & NS & NS & Usual practice & Thai & Thailand \\
\hline $\begin{array}{l}\text { Simons-Morton } \\
\text { et } \mathrm{a}^{49}\end{array}$ & Going Places & C-RCT & 11 & 57 & 18 & 36 & Teachers & NS & $72 \%$ White & USA \\
\hline $\begin{array}{l}\text { Spoth et al } \\
\text { (ISFP) }^{50}\end{array}$ & $\begin{array}{l}\text { lowa Strengthening } \\
\text { Families Program }\end{array}$ & C-RCT & 11 & 55 & 7 & 1 (day) & Project staff & $\begin{array}{l}4 \text { mailed } \\
\text { booklets on } \\
\text { changes in } \\
\text { adolescents }\end{array}$ & NS & USA \\
\hline $\begin{array}{l}\text { Spoth et al } \\
(\text { PDFY })^{50}\end{array}$ & $\begin{array}{l}\text { Preparing for the } \\
\text { Drug Free Years } \\
\text { Program }\end{array}$ & C-RCT & 11 & 55 & 5 & NS & Project staff & $\begin{array}{l}4 \text { mailed } \\
\text { booklets on } \\
\text { changes in } \\
\text { adolescents }\end{array}$ & NS & USA \\
\hline $\begin{array}{l}\text { Spoth et al } \\
(\text { LST }+ \text { SFP })^{51}\end{array}$ & SFP 10 & C-RCT & 12.5 & 45 & $\begin{array}{l}7 \times 1 \mathrm{~h}+4 \\
\text { boosters }\end{array}$ & $\begin{array}{l}1 \text { (day) + boosters } \\
1 \text { yr later }\end{array}$ & $\begin{array}{l}\text { Project staff } \\
\text { and teachers }\end{array}$ & NS & $95 \%+$ White & USA \\
\hline Spoth et al (LST) & SFP 10 & $\mathrm{C}-\mathrm{RCT}$ & 12.5 & 45 & $15 \times 45 \mathrm{~min}$ & NS & $\begin{array}{l}\text { Project staff } \\
\text { and teachers }\end{array}$ & NS & $95 \%+$ White & USA \\
\hline Storr et $a p^{2}$ & & C-RCT & 5.7 & 47 & NS & NS & Teachers & Usual practice & $\begin{array}{l}86 \% \\
\text { African-American }\end{array}$ & USA \\
\hline $\begin{array}{l}\text { Telch et al } \\
\text { (no peers) }\end{array}$ & & C-RCT & 12 & 47 & 5 & 0.75 & Teachers & No curriculum & 24\% White & USA \\
\hline $\begin{array}{l}\text { Telch } 1990 \\
\text { (peers) }\end{array}$ & & C-RCT & 12 & 47 & 5 & 0.75 & Peers & No curriculum & $24 \%$ White & USA \\
\hline $\begin{array}{l}\text { Unger et al } \\
\text { (CHIPS) }^{54}\end{array}$ & $\begin{array}{l}\text { Choosing Healthy } \\
\text { Influences for a } \\
\text { Positive Self }\end{array}$ & C-RCT & 11 & 54 & NS & NS & $\begin{array}{l}\text { Health } \\
\text { educators }\end{array}$ & Usual practice & $61 \%$ Hispanic & USA \\
\hline
\end{tabular}




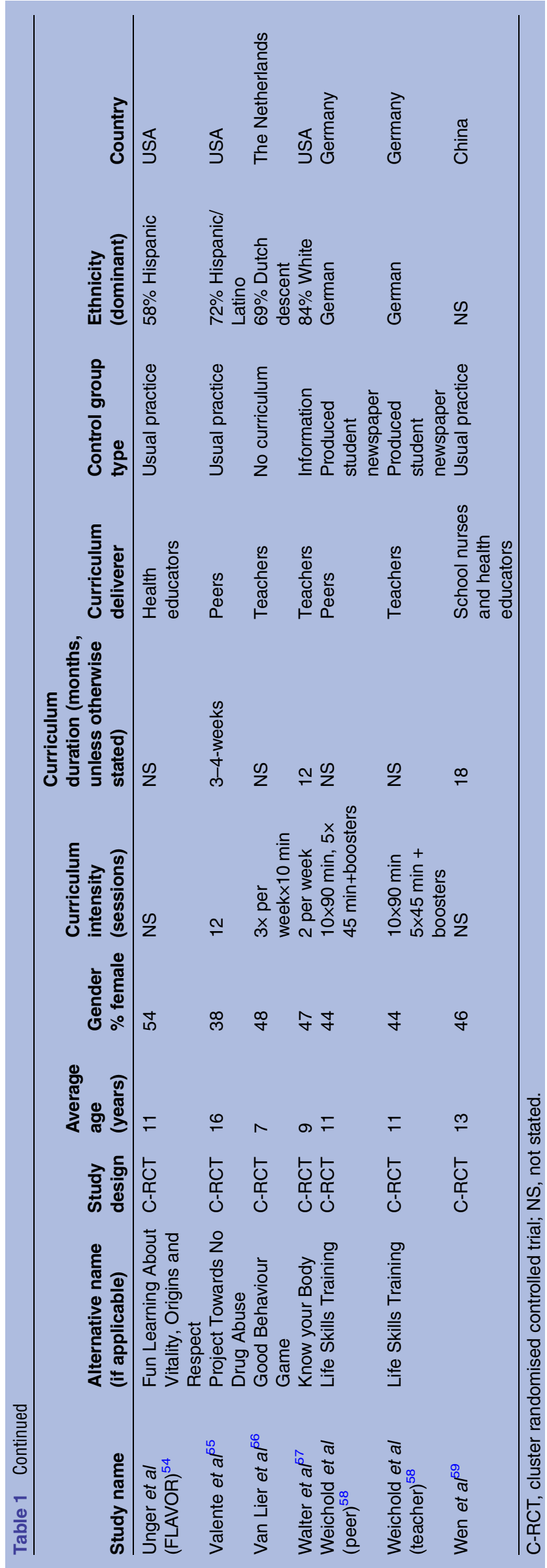

Furthermore, at 1 year or less follow-up duration, sensitivity analysis restricted to trials at low risk of selection bias $(n=12)$ showed no difference from the principal findings; though, similarly, trials of combined social competence and social influences curricula no longer showed a significant result (OR 0.55, CI 0.28 to 1.10). However, longest follow-up analyses showed sensitivity to selection bias. For all trials classified as low risk of selection bias, the overall effect was no longer significant (OR 0.92, CI 0.83 to 1.01). By curricula type social competence as well as combined social competence and social influences were no longer significant, and the group of multimodal trials now favoured the control groups (OR 1.26, CI 0.78 to 2.04). Full details of the risk of bias assessments can be found in the Cochrane review. ${ }^{60}$

\section{Publication bias}

A funnel plot of all included studies did not suggest publication bias.

\section{Subgroup analyses}

(See online supplementary material D for subgroup analyses).

Gender: At 1 year, for the limited number of trials that presented data by gender, there was a statistically significant effect for females (five trials, seven arms, OR 0.68, CI 0.50 to $0.93 ; \mathrm{I}^{2}=0 \%$ ) and no significant effect for males (four trials, six arms, OR 0.76, CI 0.53 to 1.10 ; $\mathrm{I}^{2}=51 \%$ ). The largest effect was found in one trial, ${ }^{25}$ which tested a multimodal curriculum in males (OR 0.32, CI 0.16 to $0.65)$. At longest follow-up, the results were similar; statistically significant differences were found for females (seven trials, nine arms, OR 0.80, CI 0.66 to 0.97) whereas results were not statistically significant for males (six trials, eight arms, OR 0.93 , CI 0.76 to 1.15 ).

Adult-led versus peer-led: for adult-led curricula with follow-up $\leq 1$ year (21 trials, $30 \mathrm{arms}$ ), there were no significant effects except for combined social competence/ social influences curricula, which were more effective than controls (OR 0.58 , CI 0.40 to $0.85 ; \mathrm{I}^{2}=0 \%$ ). For the peer-led curricula (six trials, eight arms) compared with controls there was no overall effect, though it should be noted that social influences interventions were only tested with a single trial $^{14}$ that offered a combined social competence/social influences curriculum.

In contrast, at longest follow-up there were significant overall effects for adult-led interventions (42 trials, 57 arms) compared with the control groups (OR 0.87, CI 0.81 to $0.94 ; \mathrm{I}^{2}=23 \%$ ), and significant effects for two of the four curricula tested: social competence (five trials, seven arms, OR 0.62 , CI 0.40 to $0.96 ; \mathrm{I}^{2}=0 \%$ ) and combined social competence/social influences (seven trials, eight arms, OR 0.58 , CI 0.42 to $0.82 ; \mathrm{I}^{2}=0 \%$ ), but not for social influences or multimodal curricula. For peer-led programmes ( 8 trials, $11 \mathrm{arms}$ ) compared with controls there were no statistically significant differences overall, nor for the three curricula tested (social influences, combined social competence/social influences and 


\section{Study or Subgroup log[Odds Ratio] \\ Howard 1996 \\ Subtotal $(95 \% \mathrm{Cl})$ \\ Heterogeneity: Not applicable \\ Test for overall effect: $Z=0.86(P=0.39)$}

$-2.092$

1.1.2 Social influences curricula versus control

\section{Coe 1982}

Armstrong 1990 (Peer)

Armstrong 1990 (Teacher)

Ellickson 1990 (HealthEd)

Ellickson 1990 (Teen)

Telch 1990 (No peers)

Telch 1990 (Peers)

Nutbeam 1993 (FSE)

Nutbeam 1993 (FSE+SAM)

Nutbeam 1993 (SAM)

De Vries 1994 (High)

De Vries 1994 (Voc)

Ennett 1994

Aveyard 1999

De Vries 2003 (UK)

Ausems 2004 (In school)

Ausems 2004 (Out School)

Garcia 2005

Chou 2006

Valente 2007 (TND)

Valente 2007 (TNDNetwork)

Buller 2008 (Australia)

Buller 2008 (USA)

Resnicow 2008 (LST)

Gabrhelik 2012

Subtotal $(95 \% \mathrm{Cl})$

Heterogeneity: $\mathrm{Chi}^{2}=28.72, \mathrm{df}=24(\mathrm{P}=0.23) ; \mathrm{I}^{2}=16 \%$

Test for overall effect: $Z=0.52(P=0.60)$
Odds Ratio SE Weight

2.4445

$0.0 \%$
$\mathbf{0 . 0 \%}$

IV, Fixed, 95\% CI

Odds Ratio

IV, Fixed, $95 \% \mathrm{CI}$

1.1.3 Combined social competence and social influences curricula versus control

Botvin 1980

Botvin 1982

Botvin 1983 (Intensive)

Botvin 1983 (LST)

Botvin 1999

Seal 2006

Resnicow 2008 (Harm Min)

Luna-Adame 2013

Subtotal $(95 \% \mathrm{Cl})$

Heterogeneity: $\mathrm{Chi}^{2}=4.02, \mathrm{df}=7(\mathrm{P}=0.78) ; \mathrm{I}^{2}=0 \%$

Test for overall effect: $Z=2.80(P=0.005)$

1.1.4 Multimodal curricula versus control

De Vries 2003 (Denmark)

De Vries 2003 (Finland)

De Vries 2003 (Portugal)

0.3436

$-0.1407$

$-0.3147$

$-0.3229$

$-0.3209$

0.9839

0.36

0.3739

0.4013

0.4026

0.7836

1.1322

0.4315

0.4347

0.4408

0.8797

1.0673

0.1963

0.1436

0.1142

0.4171

0.4594

0.5772

0.4568

1.701

1.6647

0.6343

0.7561

0.4443

0.1924

$0.12[0.00,14.87]$

$0.12[0.00,14.87]$

Simons-Morton 2005

Wen 2010

Subtotal $(95 \% \mathrm{Cl})$

$-1.5545-1.93975$
$-0.0324-1.5413-1.058$

1.058

$0.1286 \quad 3.5782$

$\begin{array}{ll}-0.9582 & 0.4636\end{array}$

0.4636

$0.3 \%$

$2.0 \%$

$1.8 \%$

$1.6 \%$

$1.6 \%$

$0.4 \%$

$0.2 \%$

$1.4 \%$

$1.4 \%$

$1.3 \%$

$0.3 \%$

$0.2 \%$

$6.7 \%$

$12.5 \%$

$19.8 \%$

$1.5 \%$

$1.2 \%$

$0.8 \%$

$1.2 \%$

$0.1 \%$

$0.1 \%$

$0.6 \%$

$0.5 \%$

$1.3 \%$

$7.0 \%$

$65.8 \%$

$0.59[0.09,4.03]$ $0.90[0.44,1.82]$

$0.57[0.28,1.19]$

$0.91[0.42,2.01]$

$0.88[0.40,1.93]$

$0.88[0.19,4.10]$

$0.23[0.02,2.07]$

$1.43[0.62,3.34]$

$1.05[0.45,2.45]$

$1.08[0.46,2.56]$

$0.99[0.18,5.56]$

$1.03[0.13,8.38]$

$0.93[0.63,1.37]$

$1.14[0.86,1.51]$

$1.06[0.85,1.33]$

$0.52[0.23,1.18]$

$0.44[0.18,1.08]$

$0.14[0.04,0.43]$

$0.90[0.37,2.21]$

$2.45[0.09,68.62]$

3.08 [0.12, 80.52]

$1.32[0.38,4.57]$

$2.34[0.53,10.30]$

$0.49[0.21,1.17]$

$1.12[0.77,1.63]$

$0.97[0.86,1.09]$
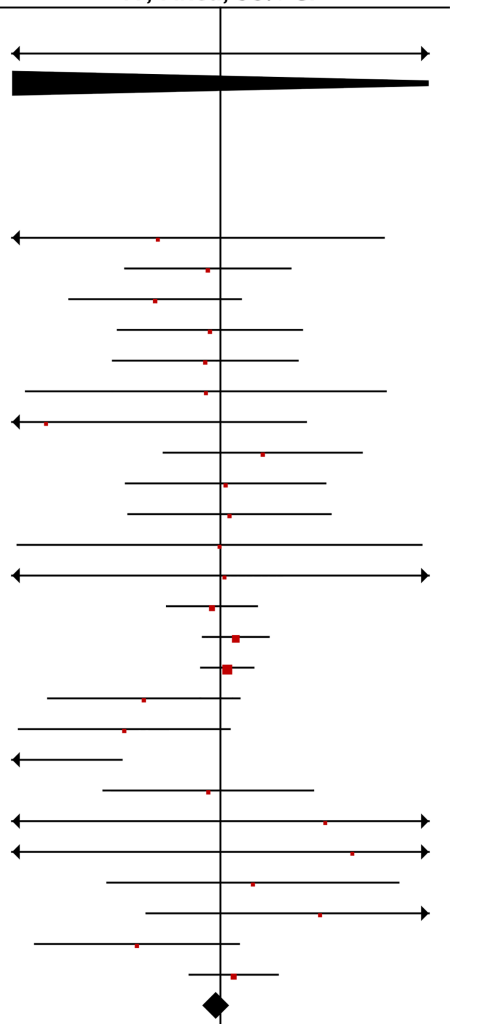

$0.1 \%$

$0.2 \%$

$0.2 \%$

$0.3 \%$

$2.1 \%$

$0.0 \%$

$1.2 \%$

$3.2 \%$

$7.4 \%$

$0.21[0.00,9.46]$

$0.97[0.11,8.39]$

$0.21[0.03,1.70]$

$0.34[0.05,2.08]$

$0.55[0.28,1.09]$

$14[0.00,1263.63]$

$0.38[0.15,0.95]$

$0.82[0.47,1.42]$

$0.59[0.41,0.85]$

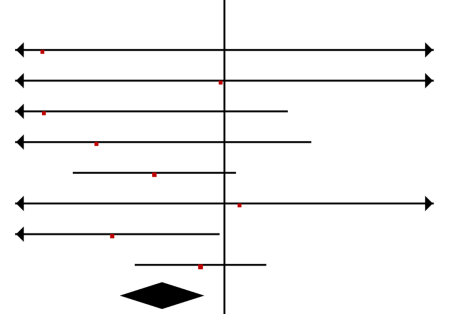

Heterogeneity: $\mathrm{Chi}^{2}=8.17, \mathrm{df}=4(\mathrm{P}=0.09) ; \mathrm{I}^{2}=51 \%$

Test for overall effect: $Z=1.31(P=0.19)$

1.1.5 Other curricula

Figa-Talamanca 1989 (F)

Figa-Talamanca 1989 (N.F)

Subtotal $(95 \% \mathrm{Cl})$

$0.1948 \quad 6.8 \%$

$0.2947 \quad 3.0 \%$

$0.1276 \quad 15.8 \%$

$0.5308 \quad 0.9 \%$

$1.0951 \quad 0.2 \%$

$26.7 \%$

$0.87[0.49,1.55]$

$0.73[0.57,0.94]$

$0.72[0.26,2.05]$

$0.73[0.08,6.21]$

$0.88[0.73,1.07]$

Figure 2 Forest plot showing results for all curricula versus control (1 year or less follow-up). 
Table 2 All curricula versus control groups, broken down by curricula type and overall

\begin{tabular}{lll}
\hline Theoretical orientation of curricula & $\begin{array}{l}\text { Curricula versus } \\
\text { control (1 year or less) } \\
\text { ORs (95\% Cl) }\end{array}$ & $\begin{array}{l}\text { Curricula versus } \\
\text { control (longest follow-up) } \\
\text { ORs (95\% Cl) }\end{array}$ \\
\hline Information only & $0.12(0.00$ to 14.87$)$ & $0.12(0.00$ to 14.87$)$ \\
Social competence & Not estimable & $0.65(0.43$ to 0.96$)$ \\
Social influences & $0.97(0.86$ to 1.09$)$ & $0.92(0.84$ to 1.00$)$ \\
Combined social competence and social influences & $0.59(0.41$ to 0.85$)$ & $0.60(0.43$ to 0.83$)$ \\
Multimodal & $0.88(0.73$ to 1.07$)$ & $0.88(0.73$ to 1.05$)$ \\
Overall & $0.91(0.82$ to 1.01$)$ & $0.88(0.82$ to 0.95$)$ \\
\hline
\end{tabular}

multimodal). Four trials that compared peer-led and adult-led interventions to controls were not included, either because it was not clear who delivered the programme $^{22} 49$ or because it was delivered online. ${ }^{18} 45$

Tobacco only versus multifocal curricula: multifocal curricula showed no overall effect compared with control either at 1 year or at longest follow-up. Multifocal social competence curriculum (five trials, seven arms, OR 0.65, CI 0.43 to $0.96 ; \mathrm{I}^{2}=0 \%$ ) and multifocal combined social competence/influences (five trials, six arms, OR 0.53, CI 0.34 to $0.83 ; \mathrm{I}^{2}=0 \%$ ) both showed a significant effect at longest follow-up. Curricula focused on only tobacco compared with control (16 trials, 27 arms) showed no effect for follow-up $\leq 1$ year (OR 0.93, CI 0.83 to 1.04; $\mathrm{I}^{2}=31 \%$ ), but there was an effect at longest follow-up (28 trials, 43 arms, OR 0.89 , CI 0.81 to $0.97 ; I^{2}=24 \%$ ). None of the other three curricula (social influences, combined social competence/social influences and multimodal) found significant differences at follow-up of either $\leq 1$ year or longest follow-up.

Adding booster sessions after the main curriculum: six trials had $3,^{28} 29464,{ }^{51} 8^{15}$ and $10^{16}$ booster sessions ranging from 1 to 2 years after the initial curricula.

Curricula without booster sessions showed no significant effect at follow-up $\leq 1$ year (24 trials, 37 arms) compared with controls (OR 0.92 , CI 0.83 to $1.02 ; \mathrm{I}^{2}=21 \%$ ), but did show significant effect at longest follow-up (45 trials, 67 arms, OR 0.90, CI 0.83 to 0.96 ; $\mathrm{I}^{2}=10 \%$ ). Similarly, for all curricula with booster sessions there were no significant differences from controls at 1 year or less (three trials, four arms, OR 0.70 , CI 0.40 to $1.07 ; \mathrm{I}^{2}=0 \%$ ), but at longest follow-up (six trials, seven arms) there was a significant difference (OR 0.73 , CI 0.55 to $0.97 ; \mathrm{I}^{2}=21 \%$ ). The combined social competence/social influences curricula, with booster sessions, had a positive effect at 1 year or less (OR 0.50 , CI 0.26 to $0.96 ; \mathrm{I}^{2}=0 \%$ ) and also at longest follow-up (OR 0.56 , CI 0.33 to $0.96 ; \mathrm{I}^{2}=0 \%$ ), but only for two ${ }^{15} 16$ and three trials, ${ }^{151651}$ respectively.

Age: an exploratory scatter plot of all trials of age versus odds ratios showed no trend and no subanalysis was completed by age.

\section{DISCUSSION}

C-RCTs with follow-up of a year or less demonstrated no overall significant effect, and the only individual curricula types that showed positive results within this group were the combined social competence/social influences curricula. The pooled results of the trials of all curricula at longest follow-up showed a positive effect in preventing starting smoking (OR $0.88,95 \%$ CI 0.82 to $0.95)$. This represents an average reduction of $12 \%$ and suggests that the effect is more evident when assessed over a longer time period. There have been no studies to identify why curricula with longer periods of follow-up are more effective.

The only individual curricula types at longest follow-up that showed a statistically significant result were social competence and combined social competence/social influence curricula.

A significant finding of this review is that over $60 \%$ of trials use social influences curricula, but these were not effective. Social influences curricula are widely used worldwide. Forty-three per cent of included trials in this review were based in the USA; here the DARE (Drug Abuse Resistance Education) programme, which is a social influences curriculum, is used in $75 \%$ of school districts. ${ }^{61}$ Few studies reported results by gender. For curricula presented by adults there were significant overall effects at longest follow-up and also for social competence and combined social competence/social influences curricula. The focus of the curricula, tobacco prevention only or multifocal, did not appear to make a difference. Pooled estimates at either 1 year or less or at longest follow-up showed estimates of a similar size. For curricula with booster sessions there was a significant effect only for combined social competence/social influences interventions with follow-up of 1 year or less and at longest follow-up.

\section{Strengths}

The strengths of the review are the comprehensive searches, use of baseline never-smoker intention-to-treat cohorts, and low heterogeneity between these trials. Comprehensive searches were conducted in multiple electronic databases, grey literature and reference lists with no limitations of date or language, and experts were consulted. It is unlikely that key trials were missed. We either derived cohorts of baseline never-smokers from trial articles or asked authors to provide such cohorts with new data runs. Using smoking outcomes from 
Figure 3 Forest plot showing results for all curricula versus control (longest follow-up).

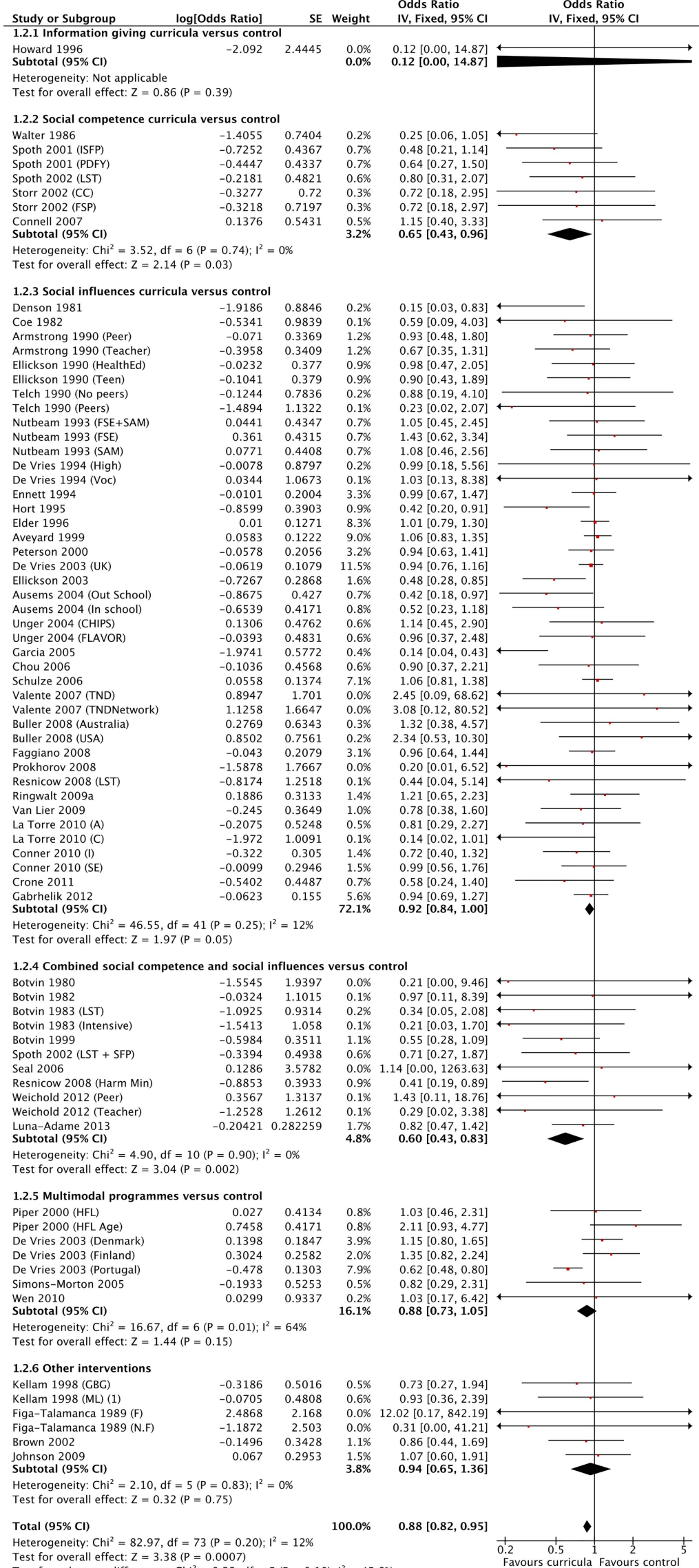

Test for overall effect: $Z=3.38(P=0.0007)$

(1) Where the figure entered remains as 0 this is because the data did not provide the absolute number for never smokers rather $s$

Favours curricula Favours control 
cohorts of baseline never-smokers provides the clearest indication of whether smoking prevention curricula are effective, and we were able to include 50 trials with 143495 baseline never-smokers. Statistical heterogeneity between these trials was low and sensitivity analyses that assessed the effects of removing studies at unclear or higher risk of bias did not change the conclusions.

\section{Limitations}

The limitations of the review are that several trials did not provide data on baseline never-smokers, some trials did not provide analysable data, and the complexity of some curricula makes them difficult to classify. It is well documented that the reporting of interventions from RCTs is poor. ${ }^{62}$ This leaves the possibility that the classification of these interventions might not be completely accurate. Nevertheless, given that all information available was extracted from the published articles, we have confidence in our classification, which reported good concordance with an independent evaluator.

We were not able to obtain baseline never-smoker data for 15 trials that reported data as changes in smoking behaviour over time, and 65 trials that provided only point prevalence of smoking data. The analyses for these trials are reported in the Cochrane review. ${ }^{60}$ From the original 256 eligible trials, we were unable to include 57 trials because authors did not provide analysable data on basic facts such as smoking outcomes or key elements of trial design (eg, n's in intervention and control groups) either in the article or by email correspondence. A further seven trials were excluded because there was no comparison to a control group or there were concerns over the data that were not resolved by email correspondence. Six trials used unique interventions that could neither be included in the prespecified five basic curricula types, nor grouped together into a sixth group.

The prespecified selection criteria were trials that compared a curriculum to a control group and we did not compare head-to-head the limited number of trials that compared curricula.

It is possible in some trials that 'never-smokers' could include some quitters, although most authors checked for inconsistencies in statements on baseline and follow-up questionnaires. Further bias could have been introduced by certain assumptions made by the review authors in data extraction, and subsequent statistical analysis. However, the consistency of results and low heterogeneity in the comparison suggest a consistent effect.

\section{Results in the context of other reviews}

This is the most systematic and comprehensive review of these curricula to date. Other reviews have considered large numbers of trials, but none have exclusively used RCTs or examined pure prevention cohorts of neversmokers. There are only three reviews published in the past 5 years, which could be expected to be up-to-date with the most recent studies and potentially comparable. However, none of them focused on assessing the effectiveness of curricula in schools to prevent smoking. Ramo et $a l^{63}$ assessed the co-use of tobacco and marijuana, Lisha and Sussman ${ }^{64}$ assessed athletic participation and tobacco and drug use, and Griffin and Botvin ${ }^{65}$ described two frequently used school curricula (Life Skills Training and Project Toward No Drug Abuse) and reviewed family and community-based programmes. Griffin provided no outcome data but concluded: "The most effective programs are highly interactive in nature, skills-focused, and implemented over multiple years." Earlier reviews are now out of date. ${ }^{66-74}$

A separate Cochrane review assessed interventions to help adolescent smokers quit. ${ }^{75}$

\section{Summary}

This review found that for baseline child and adolescent never-smokers there was no effect of school-based smoking prevention curricula with a follow-up of 1 year or less, but a $12 \%$ reduction in the onset of smoking when assessed over a longer period of follow-up. When individual curricula are considered, only social competence and combined social competence/social influences studies are effective. One interpretation why social competence interventions are effective may be that students see these as helpful to their personal development and social skills, as they provide general personal and social competence, deal with problem solving, decisionmaking, impart assertiveness and cognitive skills to resist interpersonal or media influences, teach coping strategies for stress, and provide guidance on how to increase self-control and self-esteem. There is no explanation as to why information-only, social influences $(60 \%$ of all interventions used) and multimodal curricula are not effective because no focus groups, surveys or design workshops have asked for student evaluations of their experiences with these curricula. It is possible that students perceive information curricula as lectures by adults about substance misuse.

Our review indicates that curricula delivered by adults are more effective. Adding boosters to trials with follow-up of one year or less showed no significant effect, but did at longest follow-up. Trial designers and policymakers should consider tailoring future studies to explore the various aspects of the social competence curricula with adult presenters and no booster sessions.

This review has highlighted that there are still gaps in our knowledge with regard to smoking prevention curricula. Further research is required to test curricula that would be effective for both genders. We noted that over $50 \%$ of trials were from North America and that there were limited trials exploring curricula for different ethnic groups. This would suggest that our results may reflect and be more applicable to developed countries rather than developing countries. A limited number of trials used the Internet to deliver curricula; future trials should incorporate the cultural world of adolescents (internet, media, music and teen idols). Future research needs to tailor study design to address these areas. 
Methodologically, the next steps in research are to standardise the trial design, definitions of smoking status and the content of interventions, so that more studies examine pure baseline never-smokers. Standardisation of key study design features could enable more reliable research into curricula intensity and duration (optimum number, length and frequency of sessions). Researchers should seek to utilise checklists that improve the quality of reporting ${ }^{62}$ and increase the potential impact of study findings. There is minimal information on the costs of developing and implementing these programmes and this is important as many programmes have not proven to be effective. Policymakers need to implement only curricula with proven effectiveness, and fund research projects that meet the above standardisation criteria.

Acknowledgements The authors are grateful to Jamie Hartmann-Boyce for comments on the final draft.

Contributors RET conceived the review. RET and JM completed screening and extraction of data. RP devised the analysis plan, and RET and JM provided statistical support and meta-analyses. RET and JM wrote the text. RET, JM and RP approved the final version of the review.

Funding This research received no specific grant from any funding agency in the public, commercial or not-for-profit sectors.

Competing interests None.

Provenance and peer review Not commissioned; externally peer reviewed.

Data sharing statement Data used to develop the tables and figures presented in this article are fully available in the original Cochrane review: RET, JM, RP. School-based programmes for preventing smoking. Cochrane Database of Systematic Reviews 2013, Issue 4. Art. No.: CD001293. D0I: 10.1002/14651858.CD001293.pub3.

Open Access This is an Open Access article distributed in accordance with the Creative Commons Attribution Non Commercial (CC BY-NC 4.0) license, which permits others to distribute, remix, adapt, build upon this work noncommercially, and license their derivative works on different terms, provided the original work is properly cited and the use is non-commercial. See: http:// creativecommons.org/licenses/by-nc/4.0/

\section{REFERENCES}

1. Jha P, Peto R. Global effects of smoking, of quitting, and of taxing tobacco. N Engl J Med 2014;370:60-8.

2. Epstein JA, Griffin KW, Botvin GJ. A model of smoking among inner-city adolescents: the role of personal competence and perceived social benefits of smoking. Prev Med 2000;31:107-14.

3. Centers for Disease Control and Prevention. Youth Risk Behavior Surveillance-United States, 2007. Morbidity and Mortality Weekly Report. June 6, 2008;57(SS-04).

4. NHS Information Centre (2012). Smoking, drinking and drug use among young people in England. http://www.ash.org.uk/files/ documents/ASH 108.pdf (accessed 11 Apr 2014).

5. Villanti AC, McKay HS, Abrams DB, et al. Smoking-cessation interventions for U.S. young adults. A systematic review. Am J Prev Med 2010;39:564-74.

6. Tobler NS. Lessons learned. J Prim Prev 2000;20:261-74.

7. Review Manager (RevMan) [Computer program]. Version 5.2. Copenhagen: The Nordic Cochrane Centre, The Cochrane Collaboration, 2012

8. Higgins JPT, Green S. eds. Cochrane handbook for systematic reviews of interventions version 5.1.0 [updated March 2011]. The Cochrane Collaboration, 2011. http://www.cochrane-handbook.org

9. Higgins JP, Thompson SG, Deeks JJ, et al. Measuring inconsistency in meta-analyses. BMJ 2003;7414:557-60.

10. Armstrong BK, de Klerk $\mathrm{NH}$, Shean RE, et al. Influence of education and advertising on the uptake of smoking by children. Med J Aust 1990;152:117-24.
11. Ausems M, Mesters I, Van Breukelen G, et al. Effects of in-school and tailored out-of-school smoking prevention among Dutch vocational school students. Health Educ Res 2004;19:51-63.

12. Aveyard $\mathrm{P}$, Cheng $\mathrm{KK}$, Almond J, et al. Cluster randomised controlled trial of expert system based on the transtheoretical ("stages of change") model for smoking prevention and cessation in schools. BMJ 1999;319:948-53.

13. Botvin GJ, Eng A. A comprehensive school-based smoking prevention program. J Sch Health 1980;50:209-13.

14. Botvin GJ, Eng A. The efficacy of a multi component approach to the prevention of cigarette smoking. Prev Med 1982;11:199-211.

15. Botvin GJ, Renick NL, Baker E. The effects of scheduling format and booster sessions on a broad-spectrum psychosocial approach to smoking prevention. J Behav Med 1983;6:359-79.

16. Botvin GJ, Griffin KW, Diaz T, et al. Smoking initiation and escalation in early adolescent girls: one-year follow-up of a school-based prevention intervention for minority youth. J Am Med Womens Assoc 1999;54:139-43, 152.

17. Brown KS, Cameron R, Madill C, et al. Outcome evaluation of a high school smoking reduction intervention based on extracurricular activities. Prev Med 2002;35:506-10.

18. Buller DB, Borland R, Woodall WG, et al. Randomized trials on Considers This, a tailored, internet-delivered smoking prevention program for adolescents. Health Educ Behav 2008;35:260-81.

19. Chou CP, Li Y, Unger JB, et al. A randomised intervention of smoking for adolescents in urban Wuhan, China. Prev Med 2006;42:280-5.

20. Coe RM, Crouse E, Cohen JD, et al. Patterns of change in adolescent smoking behavior and results of a one year follow-up of a smoking prevention program. J Sch Health 1982;52:348-53.

21. Connell AM, Dishion TJ, Yasui M, et al. An adaptive approach to family intervention: linking engagement in family centred intervention to reductions in adolescent problem behavior. J Consult Clin Psychol 2007;75:568-79.

22. Conner M, Higgins AR. Long-term effects of implementation intentions on prevention of smoking uptake among adolescents: a cluster randomised controlled trial. Health Psychol 2010;29:529-38.

23. Crone MR, Spruijt R, Dijkstra NS, et al. Does a smoking prevention program in elementary schools prepare children for secondary school? Prev Med 2011;52:53-9.

24. De Vries H, Backbier E, Dijkstra M, et al. A Dutch social influence smoking prevention approach for vocational school students. Health Educ Res 1994;9:365-74.

25. De Vries H, Mudde A, Leijs I, et al. The European Smoking Prevention Framework Approach (EFSA): an example of integral prevention. Health Educ Res 2003;18:611-26.

26. Denson R, Stretch S. Prevention of smoking in elementary schools. Can J Public Health 1981;72:259-63.

27. Elder JP, Perry CL, Stone EJ, et al. Tobacco use measurement, prediction and intervention in elementary schools in four states: the CATCH Study. Prev Med 1996;25:486-94.

28. Ellickson PL, Bell RM. Drug prevention in junior high: a multi-site longitudinal test. Science 1990;247:1299-305

29. Ellickson PL, McCaffrey DF, Ghosh Dastidar B, et al. New inroads in preventing adolescent drug use: results from a large-scale trial of Project ALERT in middle schools. Am J Public Health 2003;93:1830-6.

30. Ennett ST, Rosenbaum DP, Flewelling RL, et al. Long-term evaluation of Drug Abuse Resistance Education. Addict Behav 1994;19:113-25.

31. Faggiano F, Galanti MR, Bohrn K, et al. The effectiveness of a school-based substance abuse prevention program: EU-Dap cluster randomised controlled trial. Prev Med 2008;47:537-43.

32. Figa-Talamanca I, Modolo M. Evaluation of an anti-smoking educational programme among adolescents in Italy. Hygiene 1989;8:24-8.

33. Gabrhelik R, Duncan A, Miovsky M, et al. "Unplugged": a school-based randomised control trial to prevent and reduce adolescent substance use in the Czech Republic. Drug Alcohol Depend 2012;124:79-87.

34. García P, Fernández A, Sánchez JM, et al. Randomized controlled trial of a health prevention program against smoking in a population of secondary school students. Rev Calidad Asistencial 2005;20:4-13

35. Hort $\mathrm{W}$, Hort $\mathrm{H}$, Willers $\mathrm{R}$. Intervention study against cigarette smoking in Duesseldorf high school pupils [Interventionsstudie gegen das Zigarettenrauchen von Düsseldorfer Hauptschülern 1992-94]. Z Kardiol 1995;84:700-11.

36. Howard JK, Bindler RM, Synoground G, et al. A cardiovascular risk reduction program for the classroom. J Sch Nurs 1996;12:4-11.

37. Johnson CC, Myers L, Webber LS, et al. A school-based environmental intervention to reduce smoking among high school 
students: the Acadiana Coalition of Teens against Tobacco (ACTT). Int J Environ Res Public Health 2009;6:1298-316.

38. Kellam SG, Anthony JC. Targeting early antecedents to prevent tobacco smoking: findings from an epidemiologically based randomised field trial. Am J Public Health 1998;88:1490-5.

39. La Torre G, Chiaradia G, Monte L, et al. A randomised controlled trial of a school-based intervention to prevent tobacco use among children and adolescents in Italy. J Public Health 2010;18:533-42.

40. Luna-Adame M, Carrasco-Giménez TJ, Rueda-García M del M. Evaluation of the effectiveness of a smoking prevention program based on the 'Life Skills Training' approach. Health Educ Res 2013;28:673-82.

41. Nutbeam D, Macaskill $P$, Smith $C$, et al. Evaluation of two school smoking education programmes under normal classroom conditions. BMJ 1993;306:102-7.

42. Peterson AV Jr, Kealey KA, Mann SL, et al. Hutchinson Smoking Prevention Project: long-term randomised trial in school-based tobacco use prevention-results on smoking. J Natl Cancer Inst 2000;92:1979-91.

43. Piper DL, Moberg DP, King MJ. The healthy for life project: behavioral outcomes. J Prim Prev 2000;21:47-73.

44. Prokhorov AV, Kelder SH, Shegog R, et al. Impact of A Smoking Prevention Interactive Experience (ASPIRE), an interactive, multimedia smoking prevention and cessation curriculum for culturally diverse high-school students. Nicotine Tob Res 2008;10:1477-85.

45. Resnicow K, Reddy SP, James S, et al. Comparison of two school-based smoking prevention programs among South African high school students: results of a randomised trial. Ann Behav Med 2008;36:231-43.

46. Ringwalt CL, Clark HK, Hanley S, et al. Project ALERT: a cluster randomized trial. Arch Pediatr Adolesc Med 2009;163:625-32.

47. Schulze A, Mons U, Edler L, et al. Lack of sustainable prevention effect of the "Smoke-Free Class Competition" on German pupils. Prev Med 2006;42:33-9.

48. Seal N. Preventing tobacco and drug use among Thai high school students through life skills training. Nurs Health Sci 2006;8:164-8.

49. Simons-Morton B, Haynie D, Saylor K, et al. The effects of the Going Places Program on early adolescent substance use and antisocial behavior. Prev Sci 2005;6:187-97.

50. Spoth RL, Redmond C, Shin C. Randomized trial of brief family interventions for general populations: adolescent substance use outcomes 4 years following baseline. J Consult Clin Psychol 2001;69:627-42.

51. Spoth RL, Redmond C, Trudeau L, et al. Longitudinal substance initiation outcomes for a universal preventive intervention combining family and school programs. Psychol Addict Behav 2002;16:129-34.

52. Storr $\mathrm{CL}$, lalongo NS, Kellam SG, et al. A randomised controlled tria of two primary school intervention strategies to prevent early onset tobacco smoking. Drug Alcohol Depend 2002;66:51-60.

53. Telch MJ, Miller LM, Killen JD, et al. Social influences approach to smoking prevention: the effects of videotape delivery with and without same-age peer leader participation. Addict Behav 1990;15:21-8.

54. Unger JB, Chou CP, Palmer PH, et al. Project FLAVOR: 1-year outcomes of a multicultural, school-based smoking prevention curriculum for adolescents. Am J Public Health 2004;94:263-5.

55. Valente TW, Ritt-Olson A, Stacy A, et al. Peer acceleration: effects of a social network tailored substance abuse prevention program among high-risk adolescents. Addiction 2007;102:1804-15.

56. Van Lier P, Huizink A, Crijnen A. Impact of a preventive intervention targeting childhood disruptive behavior problems on tobacco and alcohol initiation from age 10 to 13 years. Drug Alcohol Depend 2009;100:228-33.

57. Walter HJ, Hofman A, Connelly PA, et al. Coronary heart disease prevention in childhood: one-year results of a randomised intervention study. Am J Prev Med 1986;2:239-49.

58. Weichold K, Silbereisen RK. Peers and teachers as facilitators of the Life Skills Program IPSY_results from a pilot study. Sucht 2012;58:247-58.

59. Wen X, Chen W, Gans KM, et al. Two-year effects of a school-based prevention programme on adolescent cigarette smoking in Guangzhou, China: a cluster randomised trial. Int $J$ Epidemiol 2010;39:860-76.

60. Thomas RE, McLellan J, Perera R. School-based programmes for preventing smoking. School-based programmes for preventing smoking. Cochrane Database Syst Rev 2013;(4):CD001293.

61. D.A.R.E. Teaching students good decision-making skills to help them lead safe and healthy lives. http://www.dare.org/about-d-a-r-e/ (accessed 30 May 2014).

62. Hoffmann TC, Glasziou PP, Boutron I, et al. Better reporting of interventions: template for intervention description and replication (TIDieR) checklist and guide. BMJ 2014;348:g1687.

63. Ramo DE, Liu H, Prochaska JJ. Tobacco and marijuana use among adolescents and young adults: a systematic review of their use. Clin Psychol Rev 2012;32:105-21.

64. Lisha NE, Sussman S. Relationship of high school and college sports participation with alcohol, tobacco and illicit drug use: a review. Addict Behav 2010;35:399-407.

65. Griffin KW, Botvin GJ. Evidence-based interventions for preventing substance use disorders in adolescents. Child Adolesc Psychiatr Clin N Am 2010;19:505-26.

66. Bruvold WH. A meta-analysis of adolescent smoking prevention programs. Am J Public Health 1993;83:872-80.

67. Rooney BL, Murray DM. A meta-analysis of smoking prevention programs after adjustment for errors in the unit of analysis. Health Educ Q 1996;23:48-64.

68. Murray DM, Short BJ. Intraclass correlation among measures related to tobacco use by adolescents: estimates, correlates, and applications in intervention studies. Addict Behav 1997;22:1-12.

69. Tobler NS, Roona MR, Ochshorn P, et al. School- based adolescent drug prevention programs: 1998 meta-analysis. J Prim Prev 2000;20:275-336.

70. Skara S, Sussman S. A review of 25 long-term adolescent tobacco and other drug use prevention program evaluations. Prev Med 2003;37:451-74.

71. Hwang MS, Yeagley KL, Petosa R. A meta-analysis of adolescent psychosocial smoking prevention programs published between 1978 and 1997 in the United States. Health Educ Behav 2004;31:702.

72. Wiehe SE, Garrison MM, Christakis DA, et al. A systematic review of school-based prevention trials with long-term follow-up. Adolesc Health 2005;36:162-9.

73. Fletcher A, Bonell C, Hargreaves J. School effects on young people's drug use: a systematic review of intervention and observational studies. J Adolesc Health 2008;4s:209-20.

74. Davo MC, Gil-Gonzalez D, Vives-Cases C, et al. Las investigaciones sobre promocion y educacion para la salud en las etapas de infantil y primaria de la escuela espanola. Una revision de los estudios publicados entre 1995 y 2005 [Research on health education and promotion in Spanish nursery and primary schools. A systematic review of studies published between 1995 and 2005]. Gac Sanit 2008;22:658-764.

75. Stanton A, Grimshaw G. Tobacco cessation interventions for young people. Cochrane Database Syst Rev 2013;(8):CD003289. 